\title{
Gelatin Encapsulated Curcumin Nanoparticles Moderate Behavior of Human Primary Gingival Fibroblasts In Vitro
}

\author{
Mai Thi-Hoang Nguyen $\mathbb{D}^{1},{ }^{1}$ Khanh Loan Ly $\mathbb{D}^{2,3}$ Thoai Quoc Kieu $\mathbb{D}^{1},{ }^{1}$ Hiep Thi Nguyen $\mathbb{D}^{2,3}$ \\ and Nam Cong-Nhat Huynh (iD) ${ }^{1}$ \\ ${ }^{1}$ Department of Dental Basic Sciences, Faculty of Odonto-Stomatology, University of Medicine and Pharmacy at Ho Chi Minh City, \\ Ho Chi Minh City, Vietnam \\ ${ }^{2}$ Tissue Engineering and Regenerative Medicine Department, School of Biomedical Engineering, International University, Vietnam \\ ${ }^{3}$ Vietnam National University, Ho Chi Minh City, Vietnam
}

Correspondence should be addressed to Hiep Thi Nguyen; nthiep@hcmiu.edu.vn

and Nam Cong-Nhat Huynh; namhuynh@ump.edu.vn

Received 12 March 2020; Revised 8 May 2020; Accepted 26 May 2020; Published 9 July 2020

Guest Editor: Anuj Kumar

Copyright $\odot 2020$ Mai Thi-Hoang Nguyen et al. This is an open access article distributed under the Creative Commons Attribution License, which permits unrestricted use, distribution, and reproduction in any medium, provided the original work is properly cited.

\begin{abstract}
Objective. Currently, there is no study evaluating the effect of nano-curcumin on human oral cells in vitro. In this study, we developed gelatin encapsulated curcumin nanoparticles (GelCur) and cultured the primary human gingival fibroblasts (hGFs) to verify the effect of GelCur on the cellular events related to oral wound healing capacities, such as cell migration and proliferation of gingival fibroblasts. Materials and Methods. GelCur was produced by the sonoprecipitation method. Particle size, zeta potential, SEM morphological observation, entrapment efficiency, and drug loading were used to characterize new GelCur. Primary hGFs were cultured from the attached gingival tissue of healthy third molar teeth. The effect of different concentrations of GelCur on hGFs was investigated by cell toxicity assay (MTT), cell proliferation assay, and cell migration assays by scratch test and transwell migration assay. Results. The average particle size of GelCur was around $356 \mathrm{~nm}$ with a moderate zeta potential of $26.5 \mathrm{mV}$. The mean PdI value of GelCur was 0.2 , while the entrapment efficiency and drug loading of curcumin in this study were around $57 \%$ and $2.4 \%$, respectively. $\mathrm{IC}_{30}$ of GelCur on hGFs was $3.96 \mathrm{mg} / \mathrm{ml}$, while $\mathrm{IC}_{50}$ was $12,37 \mathrm{mg} / \mathrm{ml}$. More than $70 \%$ of cells were viable after 24 hours incubated with 1,2 , and $3 \mathrm{mg} / \mathrm{ml} \mathrm{GelCur}$. At the concentration of $2 \mathrm{mg} / \mathrm{ml} \mathrm{GelCur}$ virtually limited cell proliferation and migration. Conclusions. GelCur remained physically stable and did not alter cell proliferation and migration. The concentration of GelCur $<3.96 \mathrm{mg} / \mathrm{ml}$ did not cause hGF cytotoxicity. Our study showed that within appropriate doses, GelCur can be used safely for hGFs.
\end{abstract}

\section{Introduction}

With the current situation of drug resistance and abuse, natural medicines have been considered for patients. Turmeric (Curcuma longa), the major source of the curcumin (diferuloylmethane), has long been recognized for its beneficial pharmacological properties and is still being widely practiced as a noninvasive wound therapy in many cultures [1]. Traditionally, curcumin has been a popular remedy for the treatment of anti-inflammation, accelerate wound healing, and minimal scar formation. The wound healing potential of curcumin, which correlated to its biochemical effects such as anti-inflammatory, anti-infectious, and antioxidant activities, has been investigated over recent years, leading to a remarkable increase of clinical trials and publications [1-3]. Furthermore, many curcumin-based wound dressings have been developed such as PVA/Chitosan/Curcumin, PVA/$\mathrm{SA} / \mathrm{TiO}_{2}$-CUR, or sodium alginate/PVA incorporated curcumin patch for its anti-inflammatory, antioxidant, and antibacterial properties that aid in the wound healing process [4-6]. Despite the medicinal biological significances, the applications of these findings into clinical treatments have so far been impeded due to several physicochemical inherent limitations of curcumin, especially its poor absorption, 
hydrophobicity, instability, rapid metabolism, and elimination $[1,7]$. To overcome these obstacles, various curcumin formulations, including emulsified micelles, nanofibers, nanocapsules, or hydrogel, have been developed.

A variety of curcumin-based systems have been produced to resolve those problems and enhance the applications of curcumin can be referred. For instance, Slika et al. have prepared curcumin-poly (allyl amine) hydrochloride-based nanocapsules to accelerate the encapsulation and release of curcumin to enhance its effectiveness against colon cancer [8]. Mouslmani and colleagues have developed a curcumin associated poly (allylamine hydrochloride)-phosphate selfassembled nanocapsules to control curcumin release and its antioxidant activity [9]. Venkatasubbu's group has incorporated curcumin into chitosan/ $/ \mathrm{TiO}_{2}$ nanoparticles to control its release for infectious wound management [10]. Especially, the development of nanobiotechnology allows for the encapsulation of curcumin in drug delivery systems such as gelatin nanoparticles has emerged as a remedial approach [11]. Gelatin nanoparticles have been recognized as potential candidates for medical applications by owning biodegradable, biocompatible, and nontoxicity properties. Moreover, the encapsulation of drug in gelatin nanoparticles enhance drug stability and control drug release effectively [12].

Oral fibroblast, including gingival fibroblast, plays important role in oral tissue homeostatic maintenance, wound healing, and tissue repair by synthesizing and secreting various extracellular matrix (ECM) proteins, containing collagen, fibronectin, and proteoglycans [13]. To investigate the natural remedies, which are safe, effective to accelerate oral wound healing and reduce the treatment costs is necessary. However, there is no study to investigate the effect of nano-curcumin in oral cells regarding cell toxicity, proliferation, and migration. Ngo and colleagues have previously developed and characterized gelatin encapsulated curcumin nanoparticles (GelCur) to implement in transdermal drug delivery study [11]. Following up on those insights, in this study, we synthesized GelCur based on established work and cultured the primary human gingival fibroblasts to verify the effect of GelCur on the cellular events related to oral wound healing capacities, such as cell migration and proliferation of gingival fibroblasts.

\section{Materials and Methods}

2.1. Fabrication of Gelatin Encapsulated Curcumin. Curcumin powders were supplied by Shanghai Zhanyun Chemical Co. Ltd. (China). Gelatin (bovine skin, type B) and poloxamer 407 (POX 407) were obtained from Sigma-Aldrich, Inc. (USA). The method of encapsulating curcumin using gelatin followed the sonoprecipitation method in the previous study [11]. Briefly, $20 \mathrm{mg}$ curcumin and $250 \mathrm{mg}$ poloxamer 407 (POX407) were dissolved in $5 \mathrm{ml}$ methanol to form solvent phase suspension. $20 \mathrm{ml}$ of gelatin solution $1 \mathrm{wt} \%$ was prepared by dissolving gelatin powder in distilled water and stirring at $400 \mathrm{rpm}$ at $40^{\circ} \mathrm{C}$ for 1 hour. The prepared solvent phase suspension (Cur/POX407) was added dropwise into antisolvent phase (gelatin solution $1 \mathrm{wt} \%$ ) while stirring at $750 \mathrm{rpm}$. The mixture was then sonicated for 20 minutes using probe sonication (QSONICA, USA) with an amplitude of $30 \%$. The mixture was kept in an icecold water bath to maintain the temperature below $30^{\circ} \mathrm{C}$. After sonication, the mixture was left stirring at $30^{\circ} \mathrm{C}$ for 2 hours for methanol to evaporate. In the subsequent step, the mixture was centrifuged at $1000 \mathrm{rpm}$ for 30 minutes to remove the pellets, then the supernatant was collected and lyophilized at $-50^{\circ} \mathrm{C}$ for 48 hours using Labconco Freeze Dryer (USA) to obtain GelCur powders (Figure 1(a)).

\subsection{Characterization of Gelatin Encapsulated Curcumin}

2.2.1. Particle Size and Zeta Potential. The particle size and zeta potential (parameter correlates with the surface charge of nanosuspension) of GelCur were analyzed using Zetasizer Nano Series (Malvern Instrument Limited, UK). $10 \mu \mathrm{l}$ of GelCur suspension was diluted with $10 \mathrm{ml}$ methanol as dispersing solution, followed by vortex shaking prior analysis. Measurements were performed in triplicate at $25^{\circ} \mathrm{C}$.

2.2.2. Entrapment Efficiency and Drug Loading. The quantity of curcumin was determined using Ultimate 3000 HPLC (Thermo Fisher Scientific Inc., USA) followed previous studies' settings [11]. $23.5 \mathrm{mg}$ of GelCur powders (equivalent to $1 \mathrm{mg}$ of curcumin corresponding to theoretical mass) was dispersed in $25 \mathrm{ml}$ distilled water, followed by ultrasonicating at $50^{\circ} \mathrm{C}$ for 30 minutes. Then, absolute ethanol was added into the cooled down mixture and adjusted accurately to obtain $50 \mathrm{ml}$ of the total solution. $100 \mu \mathrm{l}$ of the solution was diluted with $900 \mu \mathrm{l}$ methanol for HPLC analysis. The measurement was performed three times for each sample; the entrapment efficiency (EE) and drug loading (DL) were calculated using the given equations:

$\mathrm{EE}=$ the mass of drug in nanoparticles $\times 100 \% /$ total mass of drug used in the preparation of nanoparticles

$\mathrm{DL}=$ the mass of drug in nanoparticles $\times 100 \% /$ the mass of nanoparticles

2.2.3. Morphological Observation. A scanning electron microscope (SEM, JSM-IT100, JEOL, Japan) was employed to observe the morphology of GelCur powders at $10 \mathrm{kV}$. Before SEM observation, the samples were coated with Au using the sputter-coater (Cressington Sputter Coater 108 auto).

2.3. Primary Human Gingival Fibroblast Culture. Ten third molars from seven 18 to 25 -year-old healthy volunteers were extracted as recommended by their dentist. Tissues were obtained individually as previous studies $[13,14]$. Immediately after extraction, the teeth were transferred to the lab in ice-cold storage medium Dulbecco's Modified Eagle's Medium/Ham's Nutrient Mixture F12 (DMEM/F12) supplemented with $10 \%$ FBS, $500 \mathrm{UI} / \mathrm{ml}$ penicillin, $500 \mu \mathrm{g} / \mathrm{ml}$ streptomycin (Sigma, USA). The gingival tissues attached to the cervical area were carefully removed from the tooth and rinsed twice with PBS. The tissue was minced into $1 \times 2$ $\mathrm{mm}$ pieces with a surgical blade and seeded in DMEM/F12 supplemented with $10 \%$ FBS, $1 \%$ antibiotics. The tissue samples were incubated separately at $37^{\circ} \mathrm{C}$ humidified atmosphere with $5 \% \mathrm{CO}_{2}$, the medium was replaced every 3 days until outgrowing cells reached confluence. The primary 


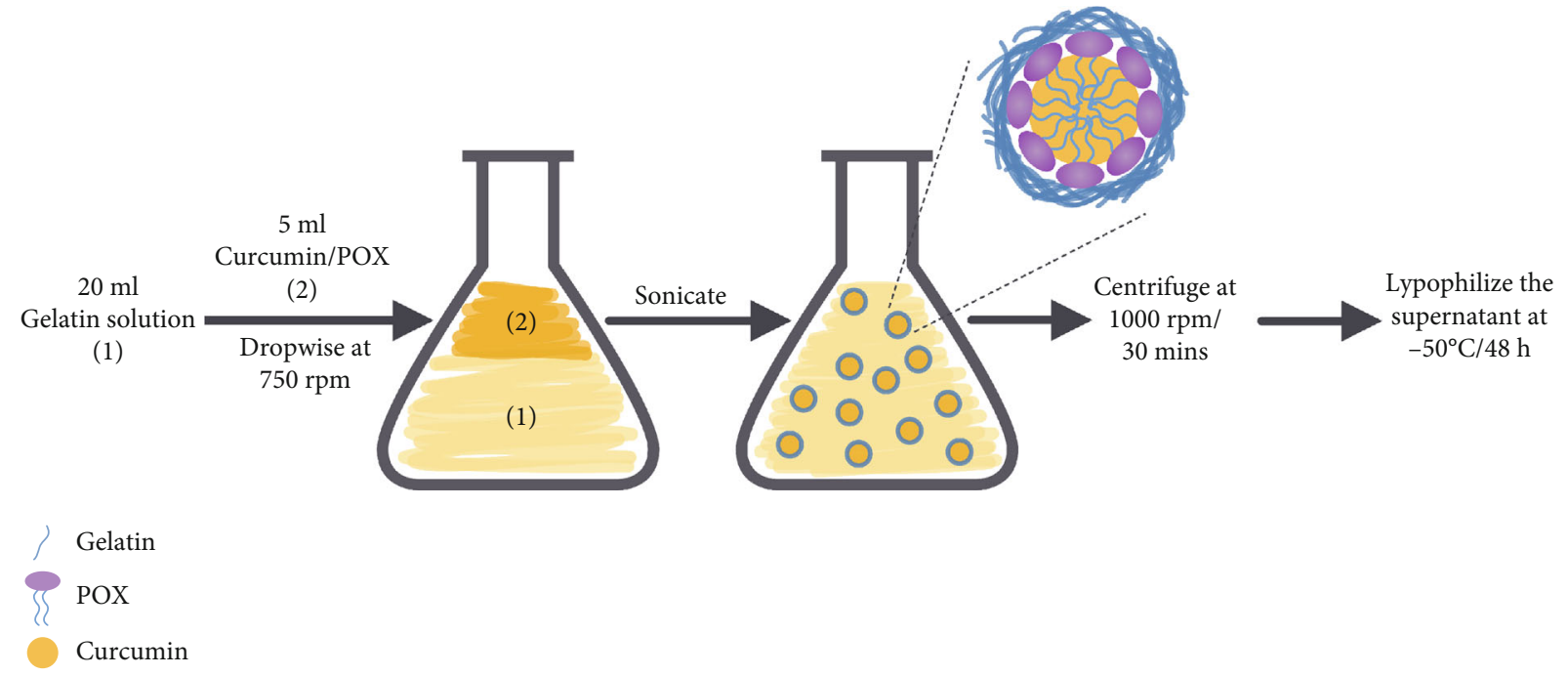

(a)
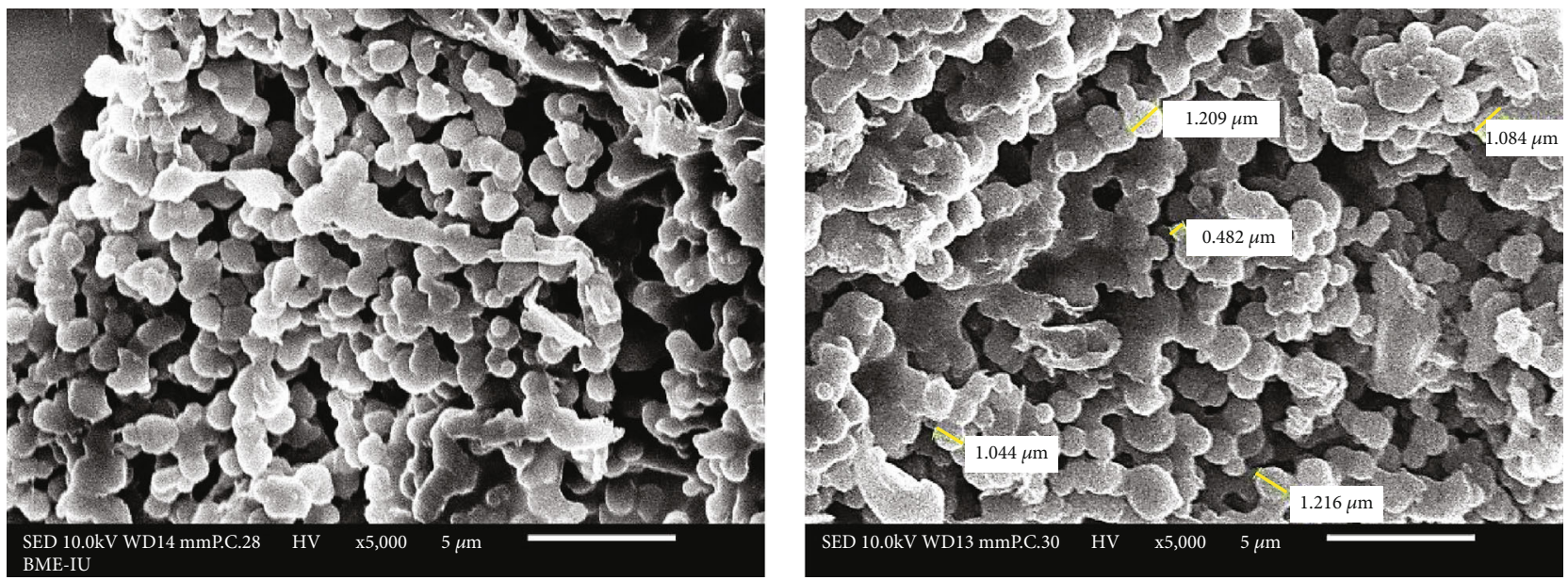

(b)

FIGURE 1: (a) Schematic of gelatin encapsulated curcumin nanoparticles (GelCur) production. (b) SEM micrographs of GelCur (left) and the nanoparticle size measurements (right) at $\times 5000$ magnification.

hGFs at the 3rd-6th passage were used for the experiments. The patients provided written informed consent for the use of discarded tissues for research purposes. Tissue samples were de-identified and analyzed anonymously. The use of gingival tissues for research purposes was approved by the Ethics Committee of the University of Medicine and Pharmacy at Ho Chi Minh City (No. 277/DHYD-HDDD).

2.4. Cell Toxicity Assay. Cell toxicity assay was assessed by $3-$ (4,5-dimethylthiazol-2-yl)-2,5-diphenyl tetrazolium bromide assay (MTT, USB Corporation, USA) $[15,16]$. The cells were plated at $10^{4}$ cells/well in 96-well plates. Cells were incubated with $1,2,3,4$, and $5 \mathrm{mg} / \mathrm{ml}$ GelCur with the approximate concentration of curcumin being 66, 132, 198, 264, and $330 \mu \mathrm{M}$, respectively (diluted in 10\% FBS DMEM/F12 and serum-free DMEM/F12). DMSO 20\% was used as the control based on the ability to induce cell death of DMSO from $20 \%$ as the previous studies [17, 18]. After $24 \mathrm{~h}$, the medium was replaced with $100 \mu \mathrm{l}$ MTT solution and incubated for $30 \mathrm{~min}$ at $37^{\circ} \mathrm{C}$. The formazan product was dissolved in solubilization/stop solution. Using a microplate reader (EZ Read 400, Biochrom, UK), the optical densities were measured at $570 \mathrm{~nm}$. The relative growth rate (RGR) as ISO 10993-5 was calculated by: $\% R G R=\left(\mathrm{OD}_{570 \mathrm{e}} / \mathrm{OD}_{570 \mathrm{~b}}\right) \times$ $100 \%$, in which OD570e is the optical density of GelCur treated group and OD570b is the optical density of $10 \%$ FBS DMEM/F12 group. Cells were toxic when \%RGR which is lower than $70 \%$ [19].

2.5. Cell Proliferation Assay. For proliferation assay, cells were plated at $10^{3}$ cells/well in 96-well plate. From the result of toxicity assay, cells were treated with a proper dose of GelCur (diluted in 10\% FBS DMEM/F12 and serum-free DMEM/F12) and control groups. After 1, 3, 5, 7, 9, 11 days, cell numbers were counted by trypan blue $0.4 \%$ as previously [16]. Briefly, $20 \mu \mathrm{l}$ of cell suspension from each well was mixed with $20 \mu$ l trypan blue solution following by 3 minutes incubation at room temperature. The cells mixture was loaded into a hemocytometer and counted by a light microscope [16]. 
2.6. Scratch-Test Assay ("wound healing assay"). Firstly, $10^{5}$ cells/well were seeded in 6-well-plate. After cell starving, a sterile $100 \mu \mathrm{l}$ pipette tip was used to make a straight scratch line on the monolayer of confluent cells at the bottom of the culture plate. The debris was washed away with PBS, and the cells were then cultured at $37^{\circ} \mathrm{C}$, humidified $5 \%$ $\mathrm{CO}_{2}$. The cells were treated with GelCur at the concentration of $2 \mathrm{mg} / \mathrm{ml}$ by diluting in DMEM (with or without FBS). Culture medium contains 10\% FBS was used as positive control, while the serum-free medium was used as the negative control. At time point $0 \mathrm{~h}$ and $24 \mathrm{~h}$, the whole cell-free areas of each well were observed and recorded by an inverted microscope (CKX53, Olympus, Japan) and digital camera in the same position. The area of "wound healing" was analyzed using the Image-Analysis J $1.45 \mathrm{~S}$ software as described previously [13].

2.7. Transwell Migration Assay. 3D cell migration assay was performed in 24-well size transwell inserts with $8.0-\mu \mathrm{m}$-pore polycarbonate membrane and $0.3 \mathrm{~cm}^{2}$ effective growth area (BD Falcon ${ }^{\mathrm{TM}}$ Cell Culture Inserts, BD Biosciences, USA). 3 $\times 105$ cells in a total of $200 \mu \mathrm{l}$ medium were seeded in each insert. To prevent cell proliferation, the migration assay was performed with serum-free medium in the presence or absence of GelCur for 16 hours. On the next day, nonmigrated cells from the upper surface of the membrane were carefully removed by a cotton swab. The cells that migrated to the other side of the membrane were fixed by formaldehyde 3,7\% in PBS for 2 minutes following by $100 \%$ methanol. Then, cells were stained with Giemsa's azur eosin methylene blue solution (Merck, Germany) and washed two times in PBS [20].

2.8. Data Analysis. All experiments were repeated with three different cell lines, each line was repeated three times. For statistical analysis, Kruskal-Wallis rank sum test and Dunn Kruskal-Wallis multiple comparisons posthoc test were used to compare between groups. We analyzed by package drc (dose-response curve), $\mathrm{R}$ version 3.6.1 with the level of significance being 0.05. $p$ values were adjusted with Bonferroni correction for multiple testing.

\section{Results}

3.1. Characteristics of GelCur. Following the production of GelCur, the analysis showed that the average particle size of GelCur was around $356 \mathrm{~nm}$ with a moderate zeta potential of $26.5 \mathrm{mV}$ (Table 1), which remained physically stable and considered to be acceptable in this study. The PdI reveals the particle size distribution of nanosuspension, where the smaller range of particle size, the smaller PdI values and the contrary. The mean PdI value of GelCur was as small as 0.2 indicating that this GelCur nanosuspension possessed a uniform size distribution for monodisperse standards.

The entrapment efficiency measures the amount of curcumin that can be loaded into nanoparticles. The entrapment efficiency and drug loading of curcumin in this study were around $57 \%$ and $2.4 \%$, respectively. The entrapment efficiency is determined by the concentration of drug carriers
TABLE 1: Characteristics of GelCur including particle size, particle size distribution (PdI), zeta potential, entrapment efficiency, and drug loading. Each measurement was repeated at least in three independent experiments (SD: standard deviation).

\begin{tabular}{lc}
\hline Parameter & Value \pm SD \\
\hline Particle size $(\mathrm{nm})$ & $356.8 \pm 35.3$ \\
PdI & $0.21 \pm 0.07$ \\
Zeta potential $(\mathrm{mV})$ & $26.5 \pm 2.1$ \\
Entrapment efficiency (\%) & $57.03 \pm 5.5$ \\
Drug loading (\%) & $2.43 \pm 0.23$ \\
\hline
\end{tabular}

(gelatin), in which the higher the concentration of gelatin, the higher the viscosity of nanosuspension. The increase in viscosity could prevent diffusion between solution and antisolvent, where the larger particles containing higher curcumin content were fabricated. SEM was also employed to confirm the morphology of GelCur powders (Figure 1(b)). Further characterizations of GelCur including Fouriertransform infrared spectroscopy, X-ray diffraction, and Cur release profile can be found in the previous study [11].

3.2. Primary Culture of hGFs. At day 4-8, cells appeared from gingiva tissues by outgrowth culture method (Figure 2(a)). These cells started with different shapes (star, round, elongated shapes) and then gradually exhibited uniformed typical fibroblast-like morphology with a large oval nucleus and many cytoplasmic tails on day 10 (Figures 2(a) and 2(c)). The cells were healthy growing and reached confluent at day 20, even at passage $6^{\text {th }}$ (Figure 2(b)).

3.3. GelCur Induces Cytotoxicity of hGFs in a Dose-Dependent Manner. To assess the cell viability, hGFs were treated with different concentrations of GelCur $(1-5 \mathrm{mg} / \mathrm{ml})$ for 24 hours, and cytotoxicity was measured by MTT assays. Results indicated that GelCur induced cytotoxicity in a dose-dependent manner (Figure 2(e)). The alteration of the shape and morphology of hGFs upon their incubation with GelCur was observed by an optical microscope (Figure 2(d)). Different concentrations of GelCur diluted in culture medium produced different shade of yellow colors. hGFs with normal morphology (spindle shape) were observed at the concentration 1,2 , and $3 \mathrm{mg} / \mathrm{ml}$ of GelCur, which are homologous with the negative control group. Meanwhile, treated with higher concentrations of GelCur ( 4 and $5 \mathrm{mg} / \mathrm{ml}$ ), some hGFs with abnormal morphologies (shrinkage) are detected. More particularly, hGFs viability was decreased to $88.8 \%, 78.7 \%$, $72.5 \%, 69.9 \%$, and $68.1 \%$ for the concentrations of $1,2,3,4$, and $5 \mathrm{mg} / \mathrm{ml}$, respectively. However, there was no significant difference within serum medium and GelCur groups by adjusted $p$ values. Only DMSO $20 \%$ gave to significant cytotoxicity on hGFs. From the $\%$ of cell viability, $\mathrm{IC}_{50}$ and $\mathrm{IC}_{30}$ of GelCur on hGF were calculated $(12,37 \mathrm{mg} / \mathrm{ml}$ and 3,96 mg/ml, respectively). According to ISO 10993-5:2009 standard for in vitro cytotoxicity, a dose of GelCur $>3.96 \mathrm{mg} / \mathrm{ml}$ which resulting in $<70 \%$ viable cells after 24 hours will be cytotoxic to hGFs [19]. 


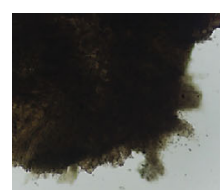

Day 1
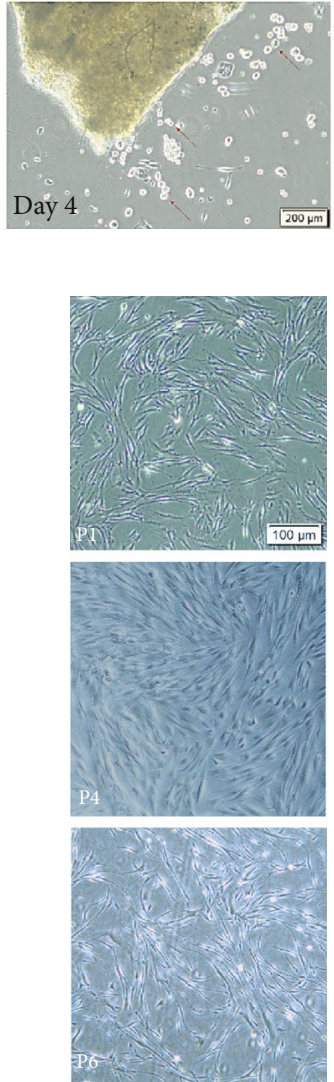

(b)
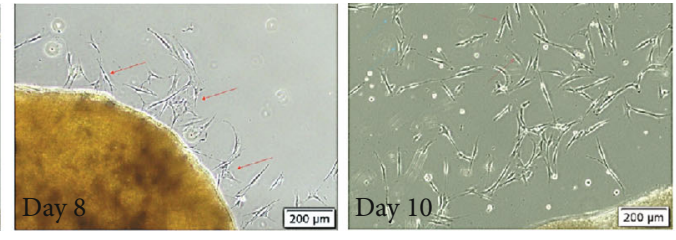

(a)

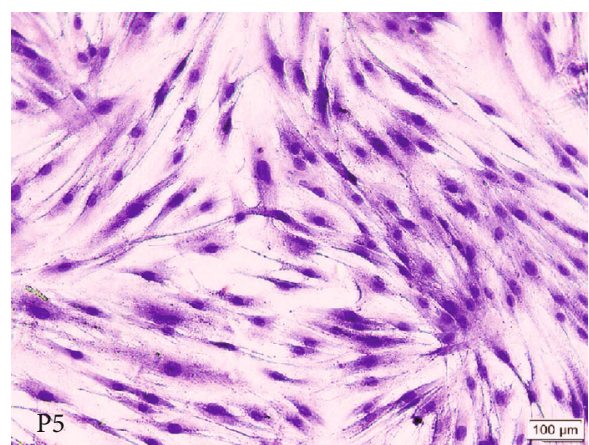

(c)
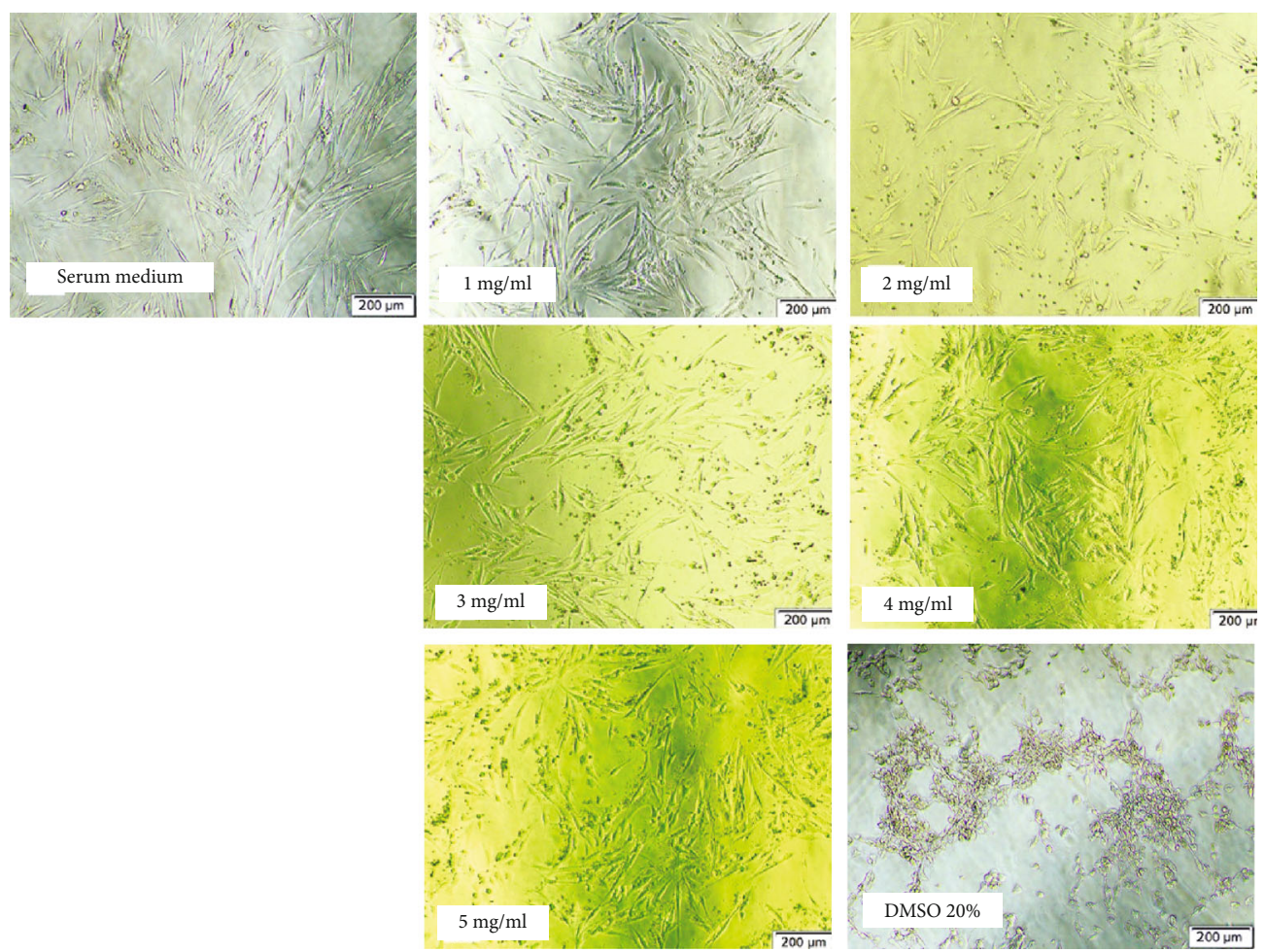

(d)

Figure 2: Continued. 


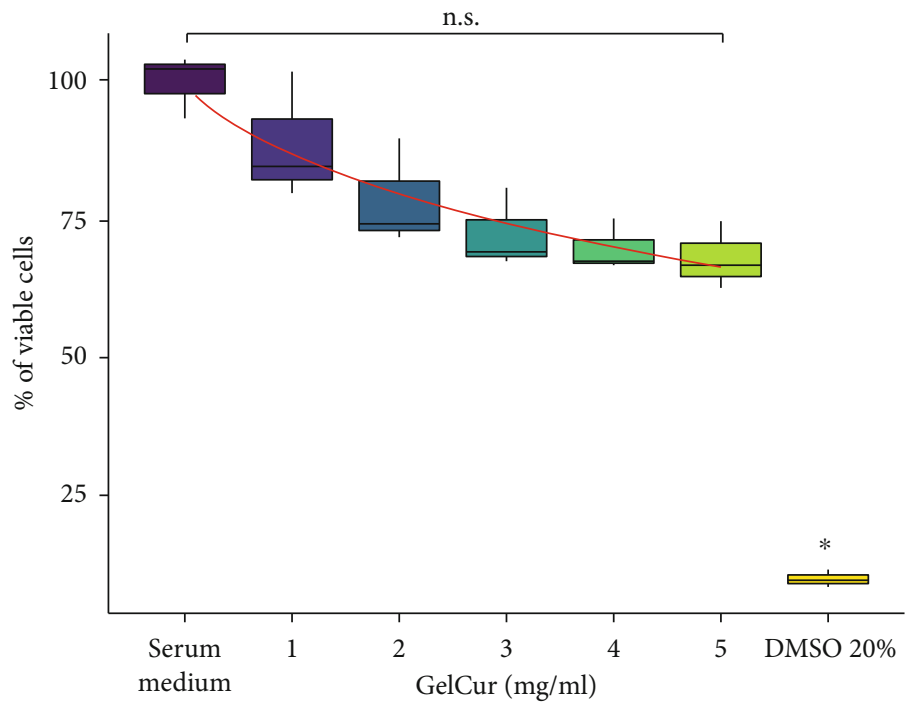

(e)

FIGURe 2: (a) Primary culture of hGFs from the gingival tissue at day 1-20. (b) hGFs at passage $1^{\text {st }}, 4^{\text {th }}$, and $6^{\text {th }}$. (c) hGF morphology by H\&E staining at passage $5^{\text {th }}$. (d) hGF culture under various conditions and concentrations of GelCur after 24 h. (e) $\%$ of hGF viability under different conditions (Dunn Kruskal-Wallis multiple comparisons, there was a significant difference between DMSO 20\% vs. serum medium group with adjusted $* p<0.05$, no significant difference was found in GelCur, serum medium groups).

3.4. GelCur Inhibits hGFs Proliferation. Base on the results of the cell toxicity assay, the concentration of $2 \mathrm{mg} / \mathrm{ml}$ was used to evaluate the effects of nano-curcumin on the proliferation of hGFs. hGFs allowed to proliferate through serum-free and serum-supplemented DMEM were used as negative and positive controls. GelCur inhibited hGFs proliferation in a time-dependent manner (Figure 3(a)). The cell number of the positive control group significantly increased and reached a peak at day 7, while a reverse trend was observed in other groups. Data indicated that from day 5 to 11 , cell proliferation was significantly (adjusted $p<0.05$ ) suppressed in both hGFs treated with GelCur in serum-free and serum medium in comparison with the positive control group. Additionally, on day 9, cell numbers in GelCur/serum medium groups were higher than GelCur/serum-free group. Generally, the proliferation of hGFs was substantially reduced after curcumin administration.

3.5. GelCur Do Not Influence the Migration of $h G F s$. To investigate the effect of nano-curcumin in hGFs migration during the oral wound-healing process, a scratch-test assay was conducted. After 24 hours, migrated cells toward the provisional gap were observed only in the serum medium group, which indicated the natural rate of cell migration (Figure 3(b)). The migrated hGFs were noted to be the lower in the presence of GelCur. However, the analysis showed no significant difference among groups (Figure 3(c)). Overall, although at the nontoxicity level, GelCur did not influence the migration of gingival fibroblasts.

3.6. GelCur Is Not a Chemoattractant Factor Moderating $h$ GFs Motility. To clarify whether nano-curcumin plays a role as a chemoattractant factor stimulating fibroblast migration, a transwell migration assay was performed. hGFs were seeded in the upper chamber, while the bottom chamber contained $2 \mathrm{mg} / \mathrm{ml}$ GelCur diluted in serum-free DMEM or $10 \%$ FBS DMEM. hGFs were allowed to migrate through the membrane in the medium with or without FBS as positive and negative controls, respectively. GelCur treatments were found to reduce the number of migrated hGFs after 16 hours (Figure 3(d)). The significant reduction was noted in hGFs incubated with $2 \mathrm{mg} / \mathrm{ml}$ GelCur in both serum-free $(2,29 \pm 1,36)$ and serumsupplemented DMEM $(3,52 \pm 1,77)$ compared with positive control group $(25,01 \pm 4,01)$ (Figure $3(\mathrm{e})$ ).

\section{Discussion}

The therapeutic applications of curcumin have been limited due to its low solubility, short half-life, and poor bioavailability. To overcome those problems, a variety of curcumin formulations have been developed including emulsified micelles, nanoformulations, or incorporating in hydrogels, microgels, and nanofibers [2, 10]. Among the possible approaches, we chose gelatin, a natural protein obtained from the hydrolysis of collagen, which has been well-known for its biodegradability, biocompatibility, and nontoxicity as curcumin carrier (emulsifier). The use of gelatin as a curcumin carrier can increase curcumin solubility, stability as well as control its release. Most importantly, gelatin has been commonly used as an effective drug carrier owing to its diverse functional groups within its backbone, which offers many benefits for chemical modification and drug attachment, giving the site-specificity for efficient drug delivery [21, 22].

Particle size and surface charge (represented as zeta potential) are among the two important characteristics of nanocomposite. They determine the drug loading, drug 


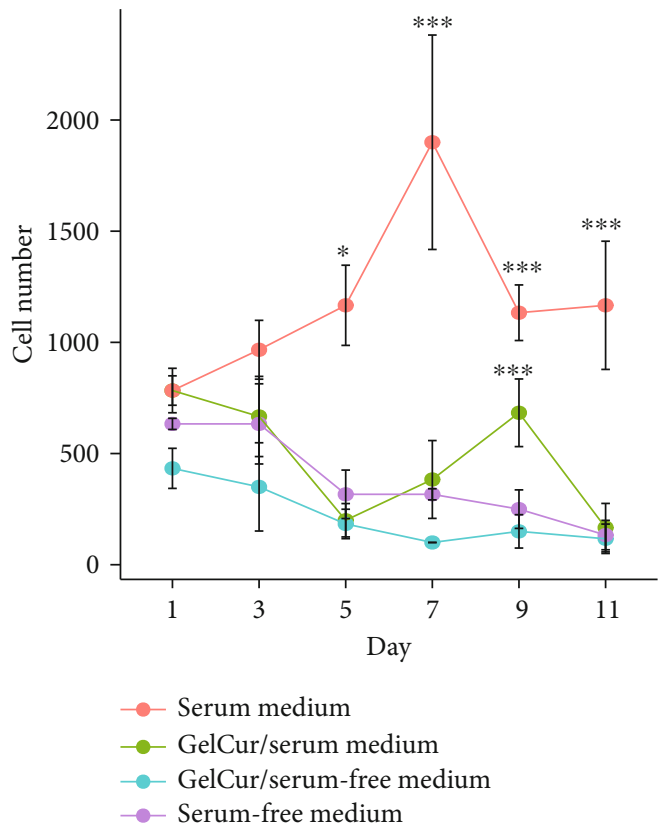

(a)

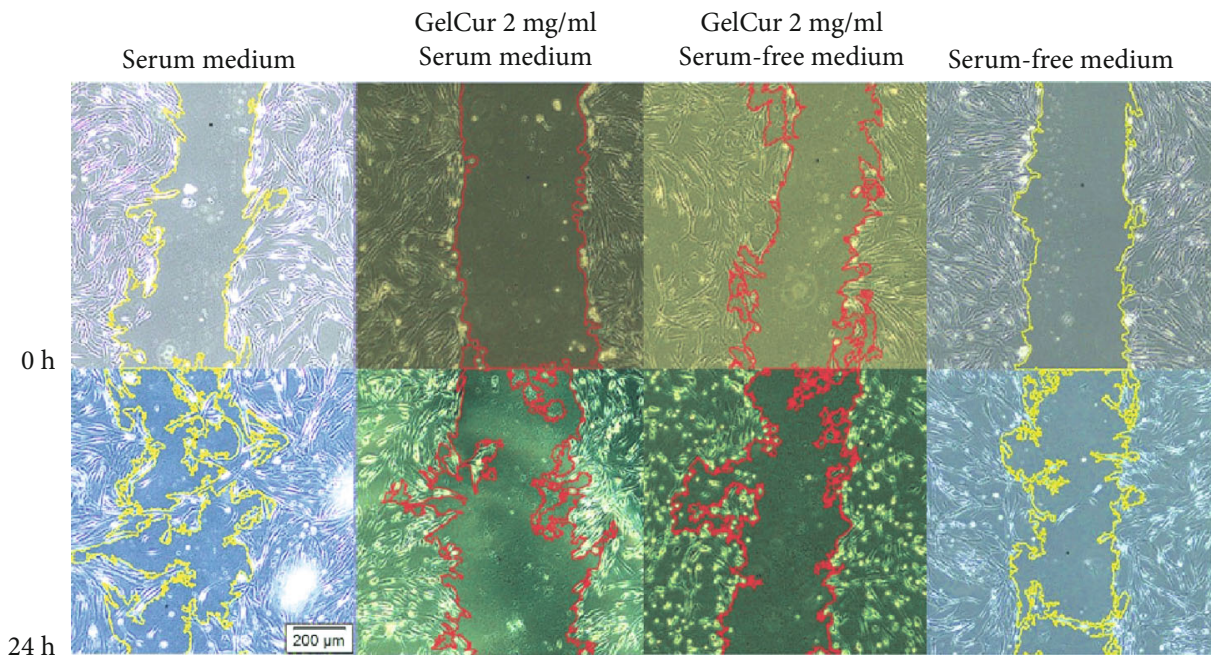

(b)

Figure 3: Continued. 


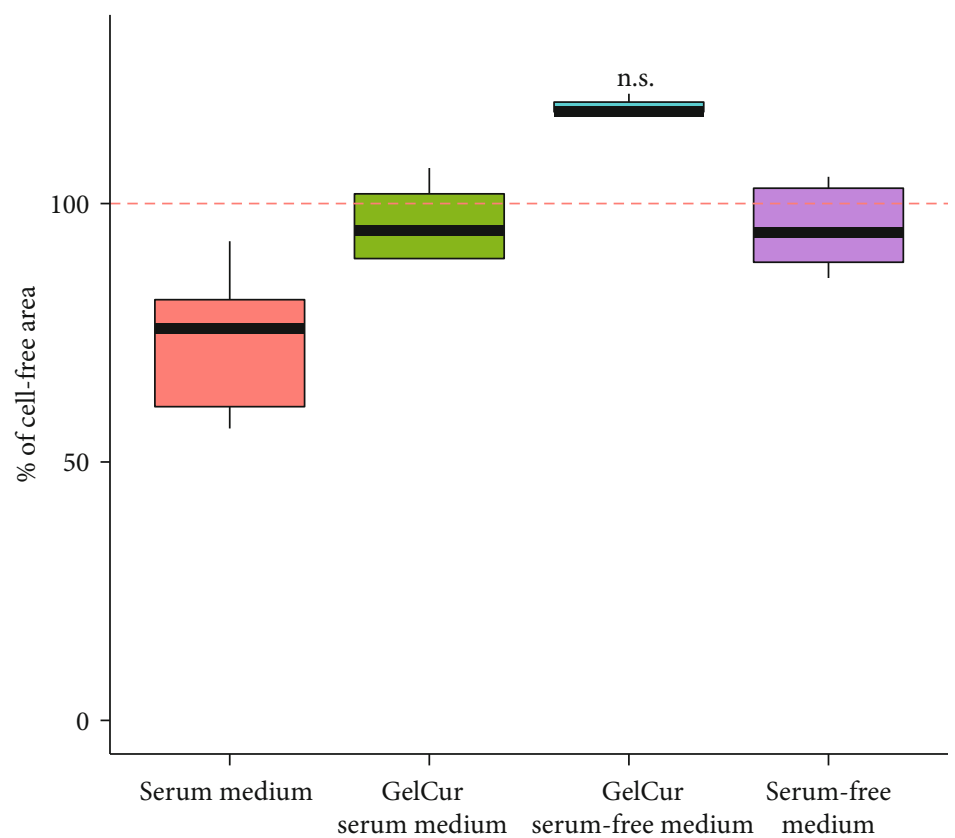

(c)

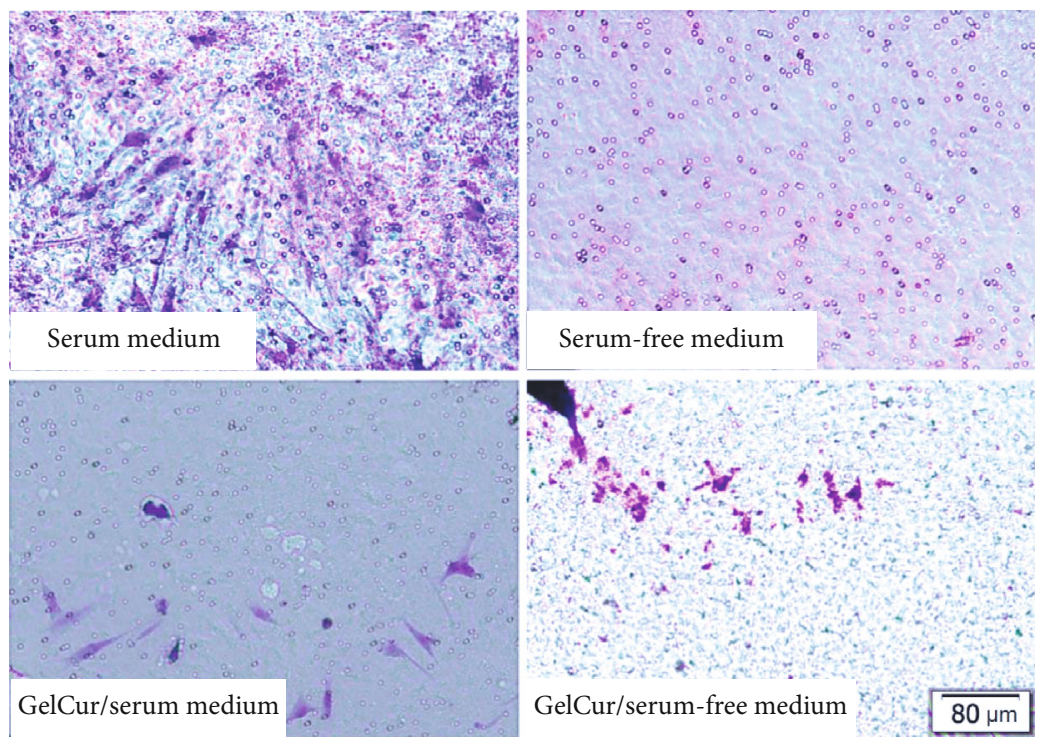

(d)

Figure 3: Continued. 


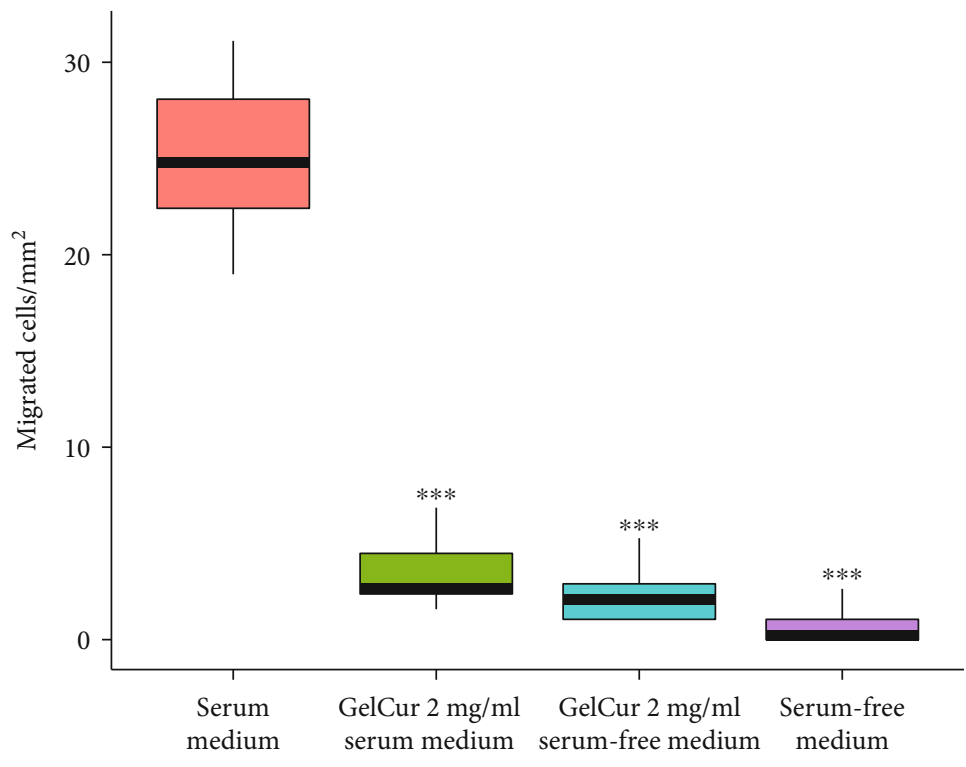

(e)

FIGURE 3: (a) Cell number of proliferation assay from day 1 to day 11 (Dunn Kruskal-Wallis multiple comparisons p-values adjusted with the Bonferroni method, there were significant differences between groups with $* p<0.05$ or $* * * p<0.001$ vs. other groups in the same day). (b) In vitro scratch test assay at $0 \mathrm{~h}$ and $24 \mathrm{~h}$. (c) \% of cell-free area after 24 hours (Kruskal-Wallis rank sum test, there was no significant difference between groups, n.s. non-significant). (d) Migrated hGFs by transwell migration assay after 16 hours. (e) Number of migrated cells in different conditions (Dunn Kruskal-Wallis multiple comparisons, there were significant differences between groups with adjusted $* * * p<0.001$ vs. serum medium group).

release, stability, and bioactivity of nanocomposite as well as influence cell-drug interactions [23]. In particular, the surface charge of near $\pm 30 \mathrm{mV}$ suggests that the GelCur nanocomposite is stable and has a low risk of particle aggregate. In terms of particle size, the size of submicroscale of GelCur particles can enhance their surface interaction while not enable direct penetration through the cell membrane. Once GelCur particles attach to the surface, Cur can slowly be released, thus, lower the unexpected toxicity caused by high concentrations of Cur as reported previously [24]. Due to the smaller particle size than curcumin and improved drug permeation and drug release (85\% after $24 \mathrm{~h}$ ) [11], GelCur could overcome the low oral bioavailability of curcumin and increase the level and effectiveness of curcumin. On the side note, our GelCur nanosuspension produced the lyophilized-spherical particles that were slightly larger than in reported particle size results. The increase in particle size could be due to the pressure and stress emerged during lyophilization, which altered the interactions between particles and caused particle aggregation. Therefore, the use of lyophilization protectants should be considered in further research to prevent possible alteration of nanocomposite properties $[25,26]$.

Toxicity of nanomaterials is a common concern during developing a new topical wound healing agent, which is essential for clinical translation. Previous clinical trials shown that receiving high oral doses of curcumin ( $8 \mathrm{~g} /$ day) does not lead to cytotoxic concentrations due to the low oral bioavailability of curcumin [27]. However, a relatively high number of reports demonstrated that curcumin, at concentrations exerts a beneficial effect, can induce DNA damage and chro- mosomal alterations both in normal and malignant cells. Cao et al. reported $2.5-5 \mu \mathrm{g} / \mathrm{ml}$ of curcumin-induced nuclear DNA damage and apoptosis in human hepatoma G2 cells $[28,29]$. Curcumin nanoparticles were developed to increases the levels of curcumin in tissues by enhancing particle penetration [27]. Aimee et al. shown that keratinocytes treated with $5 \mathrm{mg} / \mathrm{ml}$ nano-curcumin exhibited $81.7 \%$ cell viability as compared to untreated cells $[30,31]$. Our results from our cell toxicity assay demonstrated that the survival of hGFs gradually decreased with the subsequent increase of GelCur concentration. Notably, a dose of GelCur $>3.96 \mathrm{mg} / \mathrm{ml}$ will be cytotoxic to hGFs. Previous in vitro studies used direct curcumin leading to lower safe doses than our nano-curcumin $[28,29,31]$. Additionally, human gingival fibroblasts express a specific phenotype in extracellular matrix remodeling as mentioned $[13,32,33]$. The application of nano-curcumin and human oral fibroblasts in our study may contribute to the optimal implementation of drug delivery by nanoparticles in the oral environment. As in vitro studies do not thoroughly simulate the physiologic environment, further in vivo studies and clinical trials should be conducted for providing a complete insight into the potential toxicity of GelCur.

hGFs not only enable the maintenance of the barrier function of normal oral mucosal but also play a critical role in normal wound healing [34]. Upon wounding, cells proliferate and migrate into the wound area for synthesizing and depositing newly formed matrix components. To investigate the effect of GelCur on the cellular events of hGFs related to oral wound healing capacity, including proliferation and migration, the nontoxicity concentration of GelCur $(2 \mathrm{mg} / \mathrm{ml})$ was used. 
Our data showed that the proliferation of hGFs was substantially reduced after GelCur administration. The results may explain the ability of curcumin to control the wound healing process and prevent scar formation. Nanoformulated curcumin inhibited the growth and mobility of several types of cells both in vivo and in vitro in previous studies $[35,36]$. Fibrotic progression, characterized by fibroblast proliferation and the deposition of collagens, has been effectively reduced when treated by curcumin [37]. Most of the recent studies demonstrated that the mechanism of inhibiting fibroblast proliferation by curcumin is related to blocking the TGF- $\beta 1$ signaling cascade and upregulation of cathepsin K/L expressions [38]. Furthermore, curcumin upregulated the expression and activity of p53 which associated with the inhibition of proliferation and increases apoptosis in a variety of different cell lines [39, 40]. Our data from proliferation assay correlated well with data obtained from previous studies that curcumin exhibit an antifibrotic effect by the significantly inhibited proliferation of human fibroblast [38]. We found no evidence that GelCur influences the hGF migration, in congruence with past investigations using dermal fibroblast [30]. The reason may be because gingival fibroblasts express specific molecules involved in the regulation of inflammation and extracellular matrix remodeling in comparison to other fibroblasts. It may explain the ability of gingival wounds to heal faster with less scar formation as compared to skin wounds [13, 32, 33]. From these results, our next study will focus on the antiinflammatory and antioxidant properties of our nanocurcumin by in vitro inflammation model using LPS induced gingival fibroblasts. Additionally, more studies with shorter treatment durations of nano-curcumin on hGFs and other oral cell types need to be performed to have a comprehensive understanding of nano-curcumin.

\section{Conclusion}

In this study, gelatin encapsulated curcumin nanoparticles GelCur was developed which remained physically stable and did not cause hGF cytotoxicity at concentrations $<3.96 \mathrm{mg} / \mathrm{ml}$. Our study showed that within the nontoxicity dose, GelCur did not alter the proliferation and migration of hGFs. The results suggest that GelCur can be used safely in oral fibroblasts in vitro. Further studies can focus on the antibacterial and antioxidant properties of GelCur in the oral environment.

\section{Data Availability}

Requests for access to the data in this study should be made to the corresponding author.

\section{Ethical Approval}

The use of human gingival tissues for research purposes was approved by the Ethics Committee of the University of Medicine and Pharmacy at Ho Chi Minh City (No. 277/DHYDHDDD).

\section{Conflicts of Interest}

The authors declare that they have no conflicts of interest.

\section{Acknowledgments}

We would like to thank Associate Professor Tran Le Bao Ha, Department of Physiology and Animal Biotechnology, Laboratory of Tissue Engineering and Biomedical Materials, Faculty of Biology-Biotechnology, University of Science, Vietnam National University, Ho Chi Minh City, Viet Nam for supporting this study. This work was granted in part by The University of Medicine and Pharmacy at Ho Chi Minh City as No. 175/2018/HD-NCKH.

\section{References}

[1] S. Hewlings and D. Kalman, "Curcumin: a review of its' effects on human health," Food, vol. 6, no. 10, p. 92, 2017.

[2] C. Mohanty and S. K. Sahoo, "Curcumin and its topical formulations for wound healing applications," Drug Discovery Today, vol. 22, no. 10, pp. 1582-1592, 2017.

[3] G. D. Venkatasubbu and T. Anusuya, "Investigation on Curcumin nanocomposite for wound dressing," International Journal of Biological Macromolecules, vol. 98, pp. 366-378, 2017.

[4] R. Niranjan, M. Kaushik, J. Prakash et al., "Enhanced wound healing by PVA/Chitosan/Curcumin patches: In vitro and in vivo study," Colloids and Surfaces B: Biointerfaces, vol. 182, article 110339, 2019.

[5] R. Thamarai Selvi, A. P. S. Prasanna, R. Niranjan et al., "Metal Oxide Curcumin Incorporated polymer patches for Wound Healing," Applied Surface Science, vol. 449, pp. 603-609, 2018.

[6] R. Niranjan, M. Kaushik, R. T. Selvi et al., "PVA/SA/TiO2CUR patch for enhanced wound healing application: in vitro and in vivo analysis," International Journal of Biological Macromolecules, vol. 138, pp. 704-717, 2019.

[7] S. Bisht, G. Feldmann, S. Soni et al., "Polymeric nanoparticleencapsulated curcumin ("nanocurcumin"): a novel strategy for human cancer therapy," Journal of Nanobiotechnology, vol. 5, no. 1, p. 3, 2007.

[8] L. Slika, A. Moubarak, J. Borjac, E. Baydoun, and D. Patra, "Preparation of curcumin-poly (allyl amine) hydrochloride based nanocapsules: Piperine in nanocapsules accelerates encapsulation and release of curcumin and effectiveness against colon cancer cells," Materials Science and Engineering: C, vol. 109, p. 110550, 2020.

[9] M. Mouslmani, J. M. Rosenholm, N. Prabhakar, M. Peurla, E. Baydoun, and D. Patra, "Curcumin associated poly (allylamine hydrochloride)-phosphate self-assembled hierarchically ordered nanocapsules: size dependent investigation on release and DPPH scavenging activity of curcumin," RSC Advances, vol. 5, no. 24, pp. 18740-18750, 2015.

[10] G. D. Venkatasubbu, S. Nagamuthu, T. Anusuya et al., "TiO2nanocomposite for the controlled release of drugs against pathogens causing wound infections," Materials Research Express, vol. 5, no. 2, article 024003, 2018.

[11] H. V. Ngo, P. H. L. Tran, B. J. Lee, and T. T. D. Tran, "Development of film-forming gel containing nanoparticles for transdermal drug delivery," Nanotechnology, vol. 30, no. 41, p. $415102,2019$. 
[12] N. Sahoo, R. K. Sahoo, N. Biswas, A. Guha, and K. Kuotsu, "Recent advancement of gelatin nanoparticles in drug and vaccine delivery," International Journal of Biological Macromolecules, vol. 81, pp. 317-331, 2015.

[13] N. C.-N. Huynh, V. Everts, C. Leethanakul, P. Pavasant, and R. S. Ampornaramveth, "Rinsing with saline promotes human gingival fibroblast wound healing in vitro," PLoS One, vol. 11, no. 7, article e0159843, 2016.

[14] M. T. N. Nguyen and H. L. B. Tran, "Effect of modified bovine pericardium on human gingival fibroblasts in vitro," Cells, Tissues, Organs, vol. 206, no. 6, pp. 296-307, 2019.

[15] N. C.-N. Huynh, V. Everts, P. Pavasant, and R. S. Ampornaramveth, "Inhibition of histone deacetylases enhances the osteogenic differentiation of human periodontal ligament cells," Journal of Cellular Biochemistry, vol. 117, no. 6, pp. 13841395, 2016.

[16] N. C.-N. Huynh, S. H. Le, V. N. Doan, L. T. Q. Ngo, and H. L. B. Tran, "Simplified conditions for storing and cryopreservation of dental pulp stem cells," Archives of Oral Biology, vol. 84, pp. 74-81, 2017.

[17] H. Apdik, A. Dogan, S. Demirci, S. Aydin, and F. Sahin, "Dose-dependent effect of boric acid on myogenic differentiation of human adipose-derived stem cells (hADSCs)," Biological Trace Element Research, vol. 165, no. 2, pp. 123-130, 2015.

[18] G. Da Violante, N. Zerrouk, I. Richard, G. Provot, J. C. Chaumeil, and P. Arnaud, "Evaluation of the cytotoxicity effect of dimethyl sulfoxide (DMSO) on Caco2/TC7 colon tumor cell cultures," Biological \& Pharmaceutical Bulletin, vol. 25, no. 12, pp. 1600-1603, 2002.

[19] International Organization for Standardization ISO 10993-5:, 2009 Biological evaluation of medical devices - Part 5: Tests for in vitro cytotoxicity, International Organization for Standardization, Geneva, 2009.

[20] T. Fukuda, T. Ezawa, K. Tanaka, and K. Ito, "Peripheral occluding effects of non-absorbable membranes on ingrowth of cultured gingival connective tissue cells," Journal of Periodontology, vol. 71, no. 11, pp. 1680-1686, 2000.

[21] Z. Zhou, S. He, T. Huang et al., "Preparation of gelatin/hyaluronic acid microspheres with different morphologies for drug delivery," Polymer Bulletin, vol. 72, no. 4, pp. 713-723, 2015.

[22] H. Yan, Z. Zhou, T. Huang et al., "Controlled release in vitro of icariin from gelatin/hyaluronic acid composite microspheres," Polymer Bulletin, vol. 73, no. 4, pp. 1055-1066, 2016.

[23] S. M. A. Sadat, S. T. Jahan, and A. Haddadi, "Effects of Size and Surface Charge of Polymeric Nanoparticles on in Vitro and in Vivo Applications," Journal of Biomaterials and Nanobiotechnology, vol. 7, no. 2, pp. 91-108, 2016.

[24] S. R. Damarla, R. Komma, U. Bhatnagar, N. Rajesh, and S. M. A. Mulla, "An evaluation of the genotoxicity and subchronic oral toxicity of synthetic curcumin," Journal of Toxicology, vol. 2018, Article ID 6872753, 27 pages, 2018.

[25] W. Abdelwahed, G. Degobert, S. Stainmesse, and H. Fessi, "Freeze-drying of nanoparticles: formulation, process and storage considerations," Advanced Drug Delivery Reviews, vol. 58, no. 15, pp. 1688-1713, 2006.

[26] L. L. Khanh, N. T. Truc, N. T. Dat et al., "Gelatin-stabilized composites of silver nanoparticles and curcumin: characterization, antibacterial and antioxidant study," Science and Technology of Advanced Materials, vol. 20, no. 1, pp. 276-290, 2019.
[27] E. Burgos-Morón, J. M. Calderón-Montaño, J. Salvador, A. Robles, and M. López-Lázaro, "The dark side of curcumin," International Journal of Cancer, vol. 126, no. 7, pp. 1771-1775, 2010.

[28] J. Cao, L. Jia, H.-M. Zhou, Y. Liu, and L.-F. Zhong, "Mitochondrial and nuclear DNA damage induced by curcumin in human hepatoma G2 cells," Toxicological Sciences, vol. 91, no. 2, pp. 476-483, 2006.

[29] J. Cao, Y. Liu, L. Jia et al., "Curcumin induces apoptosis through mitochondrial hyperpolarization and mtDNA damage in human hepatoma G2 cells," Free Radical Biology \& Medicine, vol. 43, no. 6, pp. 968-975, 2007.

[30] A. E. Krausz, B. L. Adler, V. Cabral et al., "Curcumin-encapsulated nanoparticles as innovative antimicrobial and wound healing agent," Nanomedicine, vol. 11, no. 1, pp. 195-206, 2015.

[31] C. Syng-Ai, A. L. Kumari, and A. Khar, "Effect of curcumin on normal and tumor cells: role of glutathione and bcl-2," Molecular Cancer Therapeutics, vol. 3, no. 9, pp. 1101-1108, 2004.

[32] W. Mah, G. Jiang, D. Olver et al., "Human gingival fibroblasts display a non-fibrotic phenotype distinct from skin fibroblasts in three-dimensional cultures," PLoS One, vol. 9, no. 3, article e90715, 2014.

[33] F. Guo, D. E. Carter, A. Mukhopadhyay, and A. Leask, "Gingival fibroblasts display reduced adhesion and spreading on extracellular matrix: a possible basis for scarless tissue repair?," PLoS One, vol. 6, no. 11, article e27097, 2011.

[34] C. A. G. McCulloch and S. Bordin, "Role of fibroblast subpopulations in periodontal physiology and pathology," Journal of Periodontal Research, vol. 26, no. 3, pp. 144-154, 1991.

[35] S. Hosseini, J. Chamani, M. R. Hadipanah et al., "Nano-curcumin's suppression of breast cancer cells (MCF7) through the inhibition of cyclinD1 expression," Breast Cancer: Targets and Therapy, vol. 11, pp. 137-142, 2019.

[36] F. Milano, L. Mari, W. van de Luijtgaarden, K. Parikh, S. Calpe, and K. K. Krishnadath, "Nano-curcumin inhibits proliferation of esophageal adenocarcinoma cells and enhances the T cell mediated immune response," Frontiers in Oncology, vol. 3, p. 137, 2013.

[37] X. Zhou, J. Zhang, C. Xu, and W. Wang, "Curcumin ameliorates renal fibrosis by inhibiting local fibroblast proliferation and extracellular matrix deposition," Journal of Pharmacological Sciences, vol. 126, no. 4, pp. 344-350, 2014.

[38] D. Zhang, C. Huang, C. Yang et al., "Antifibrotic effects of curcumin are associated with overexpression of cathepsins $\mathrm{K}$ and L in bleomycin treated mice and human fibroblasts," Respiratory Research, vol. 12, no. 1, p. 154, 2011.

[39] E. Wawryk-Gawda, P. Chylinska-Wrzos, M. Lis-Sochocka et al., "P 53 protein in proliferation, repair and apoptosis of cells," Protoplasma, vol. 251, no. 3, pp. 525-533, 2014.

[40] H. Fu, C. Wang, D. Yang et al., "Curcumin regulates proliferation, autophagy, and apoptosis in gastric cancer cells by affecting PI3K and P53 signaling," Journal of Cellular Physiology, vol. 233, no. 6, pp. 4634-4642, 2018. 